\title{
Functional Relaxation and Guided Imagery as Complementary Therapy in Asthma: A Randomized Controlled Clinical Trial
}

\author{
C. Lahmann ${ }^{a}$ e $\quad$ M. Nickel ${ }^{f} \quad$ T. Schuster ${ }^{b} \quad$ N. Sauer ${ }^{d} \quad$ J. Ronel ${ }^{a} \quad$ M. Noll-Hussong ${ }^{a}$ \\ K. Tritt ${ }^{\mathrm{e}}$ D. Nowak ${ }^{\mathrm{c}}$ F. Röhricht ${ }^{\mathrm{g}} \quad$ T. Loew $^{\mathrm{e}}$

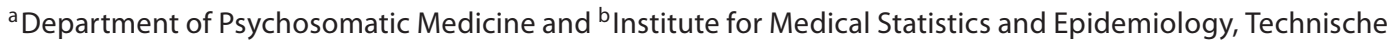 \\ Universität München, ' Institute and Outpatient Clinic for Occupational and Environmental Medicine, Ludwig \\ Maximilian University, Munich, ${ }^{\mathrm{d}}$ Department of Psychosomatic Medicine, University Medical Center \\ Hamburg-Eppendorf and Hamburg-Eilbek (Schön Clinics), Hamburg, and e Department of Psychosomatic Medicine, \\ University of Regensburg Medical Center, Regensburg, Germany; ${ }^{\mathrm{f}}$ Department of Psychosomatic Medicine, \\ Medical University of Graz, Graz, Austria; ' University of Hertfordshire and Unit for Social and Community Psychiatry, \\ Queen Mary College, University of London, London, UK
}

\section{Key Words}

Asthma · Functional relaxation · Guided imagery · Body psychotherapy $\cdot$ Randomized controlled trial

\begin{abstract}
Background: Asthma is a frequently disabling and almost invariably distressing disease that has a high overall prevalence. Although relaxation techniques and hypnotherapeutic interventions have proven their effectiveness in numerous trials, relaxation therapies are still not recommended in treatment guidelines due to a lack of methodological quality in many of the trials. Therefore, this study aims to investigate the efficacy of the brief relaxation technique of functional relaxation (FR) and guided imagery (GI) in adult asthmatics in a randomized controlled trial. Methods: $64 \mathrm{pa}-$ tients with extrinsic bronchial asthma were treated over a 4-week period and assessed at baseline, after treatment and after 4 months, for follow-up. 16 patients completed FR, 14 $\mathrm{Gl}, 15$ both $\mathrm{FR}$ and $\mathrm{Gl}$ (FR/GI) and 13 received a placebo relaxation technique as the control intervention $(\mathrm{Cl})$. The forced expiratory volume in the first second $\left(\mathrm{FEV}_{1}\right)$ as well as the specific airway resistance $\left(s R_{a w}\right)$ were employed as primary
\end{abstract}

outcome measures. Results: Participation in FR, Gl and FR/GI led to increases in $\mathrm{FEV}_{1}$ (\% predicted) of $7.6 \pm 13.2,3.3 \pm 9.8$, and $8.3 \pm 21.0$, respectively, as compared to $-1.8 \pm 11.1$ in the $\mathrm{Cl}$ group at the end of the therapy. After follow-up, the increases in FEV 1 were $6.9 \pm 10.3$ in the FR group, $4.4 \pm 7.3$ in the $\mathrm{Gl}$ and $4.5 \pm 8.1$ in the $\mathrm{FR} / \mathrm{Gl}$, compared to $-2.8 \pm 9.2$ in the $\mathrm{Cl}$. Improvements in $\mathrm{SR}_{\mathrm{aw}}$ (\% predicted) were in keeping with the changes in $\mathrm{FEV}_{1}$ in all groups. Conclusions: Our study confirms a positive effect of FR on respiratory parameters and suggests a clinically relevant long-term benefit from FR as a nonpharmacological and complementary therapy treatment option.

Copyright $\odot 2009$ S. Karger AG, Basel

\section{Introduction}

Asthma is a frequently disabling and almost invariably distressing disease that has a prevalence of between 2 and $8 \%$ in adults [1] and up to $21 \%$ in children [2]. According to Eder et al. [3], there is evidence that, in some areas of the Western world, the prevalence of asthma may have plateaued at this very high level.

\section{KARGER}

Fax +41613061234 E-Mail karger@karger.ch www.karger.com (c) 2009 S. Karger AG, Basel

0033-3190/09/0784-0233\$26.00/0

Accessible online at:

www.karger.com/pps
Claas Lahmann, MD

Department of Psychosomatic Medicine and Psychotherapy

Klinikum rechts der Isar, Technical University Munich

Langerstrasse 3, DE-81675 Munich (Germany)

Tel. +49 894140 4319, Fax +49894140 4845, E-Mail lahmann@tum.de 
Asthma is a disease of multifactorial etiology $[4,5]$. Regarding the modulation of the course of disease, Mathé and Knappe [6] have already found that psychological stress is associated with a decrease in airway resistance in healthy individuals but with an increase in those with asthma. On the one hand this presents a fundamental paradox, in as much as several components of the generic stress response (i.e. glucocorticoid release from the adrenal cortex or increased activity in the sympathetic nervous system) should in principle lead to bronchodilatation rather than bronchoconstriction. On the other hand, it shows that the idea of a generic stress response is an oversimplification and the precise psychophysiological regulation of respiratory airflow remains unclear [7].

However, since the importance of emotional factors in triggering asthma attacks has been recognized $[8,9]$, it has been suggested that psychological treatment methods may influence airway caliber by balancing the activity of the autonomous nervous system. Several psychological interventions may be employed to ameliorate health problems associated with asthma, as summarized by Yorke et al. [10]. There seem to be differential effects, with some evidence for a reduction in relief medications after relaxation therapy, improvement of asthma-related quality of life following cognitive behavioral therapy and peak expiratory flow outcome being positively influenced in biofeedback therapy [10].

The adequate management of asthmatic complaints relies heavily on the patient's ability to detect changes in breathing, to assess these changes accurately and to respond to them appropriately [11]. These considerations have led to the use of complementary relaxation techniques and body-psychotherapeutic methods for improving body awareness and better regulation of the autonomic nervous system.

A large body of literature [12] exists regarding the use of relaxation techniques in the treatment of the symptoms of asthma. These techniques include jacobsonian progressive relaxation [13], hypnotherapy [14], autogenic training [15], biofeedback training [16] and approaches based on mindfulness, such as yoga [17]. As shown in a critical review by Brown [18], hypnosis is efficacious in managing emotional states that exacerbate airway obstruction and may possibly stabilize airway hyperresponsiveness in some individuals. Freeman and Welton [19] reported that imagery interventions led to significant symptomatic improvements in adult asthmatics. Promising results have also been shown in several studies using the technique of functional relaxation (FR), a brief relaxation technique based on the principles of psychodynam- ic body psychotherapy. In two trials $[20,21]$ FR led to significant improvements in pulmonary function in asthma patients, with effect sizes comparable to those induced by inhaled $\beta_{2}$-sympathomimetics.

Despite these findings, there is still a lack of evidence regarding the efficacy of relaxation therapies in the management of asthma, which is largely due to some methodological limitations in previous studies [12]. Many of the previous trials do not fulfill the criteria of randomized controlled trials as defined by the Consort Statement [22]. Information regarding dropouts is often missing and the studies do not include a follow-up period for detecting the long-term stability of achieved short-term effects. Because of the above, relaxation and other complementary techniques are still not recommended in current asthma treatment guidelines [23].

We therefore carried out a randomized controlled trial that aimed to compare the brief relaxation technique of FR with guided imagery (GI) in adult asthmatics. In addition to the methods applied in previous studies that addressed the efficacy of FR $[20,21]$, the present trial focused on the long-term effects of FR by including a 4month follow-up assessment.

\section{Methods}

\section{Participants}

Patients suffering from allergic bronchial asthma due to dust mite allergy were recruited using a local newspaper advertisement, which was answered by 291 patients. The inclusion criterion was a diagnosis of controlled allergic asthma already established by a pulmonologist/allergologist at a university hospital outpatient clinic for respiratory diseases. Diagnostics included a positive history of dyspnea upon exposure to house dust, physical examination, lung function tests and a positive prick test to a standardized dust mite extract (Dermatophagoides pteronyssinus and/or Dermatophagoides farinae) with a wheal diameter at least $3 \mathrm{~mm}$ greater than the negative control. According to this criterion, 75 suitable patients were identified. Exclusion criteria were defined as follows: age under 18 years, severe psychiatric or somatic disease other than allergic asthma, current use of any psychoactive medication or psychotherapy (including regular relaxation training), use of medication (anything other than the usual types of asthma medication prescribed by a chest physician) or modification of asthma medication during the previous 4 weeks or within the course of the trial. Furthermore, basic asthma medication was kept stable throughout the trial.

Current mental illness was excluded using a clinical face-toface interview. Potential participants received a comprehensive description of the study specifics, and 64 patients gave their written informed consent to participate. Those included were randomized to FR, GI, FR and GI (FR/GI) or a placebo relaxation technique as the control intervention (CI) using randomized numbers generated by an Excel table (fig. 1). Randomiza- 


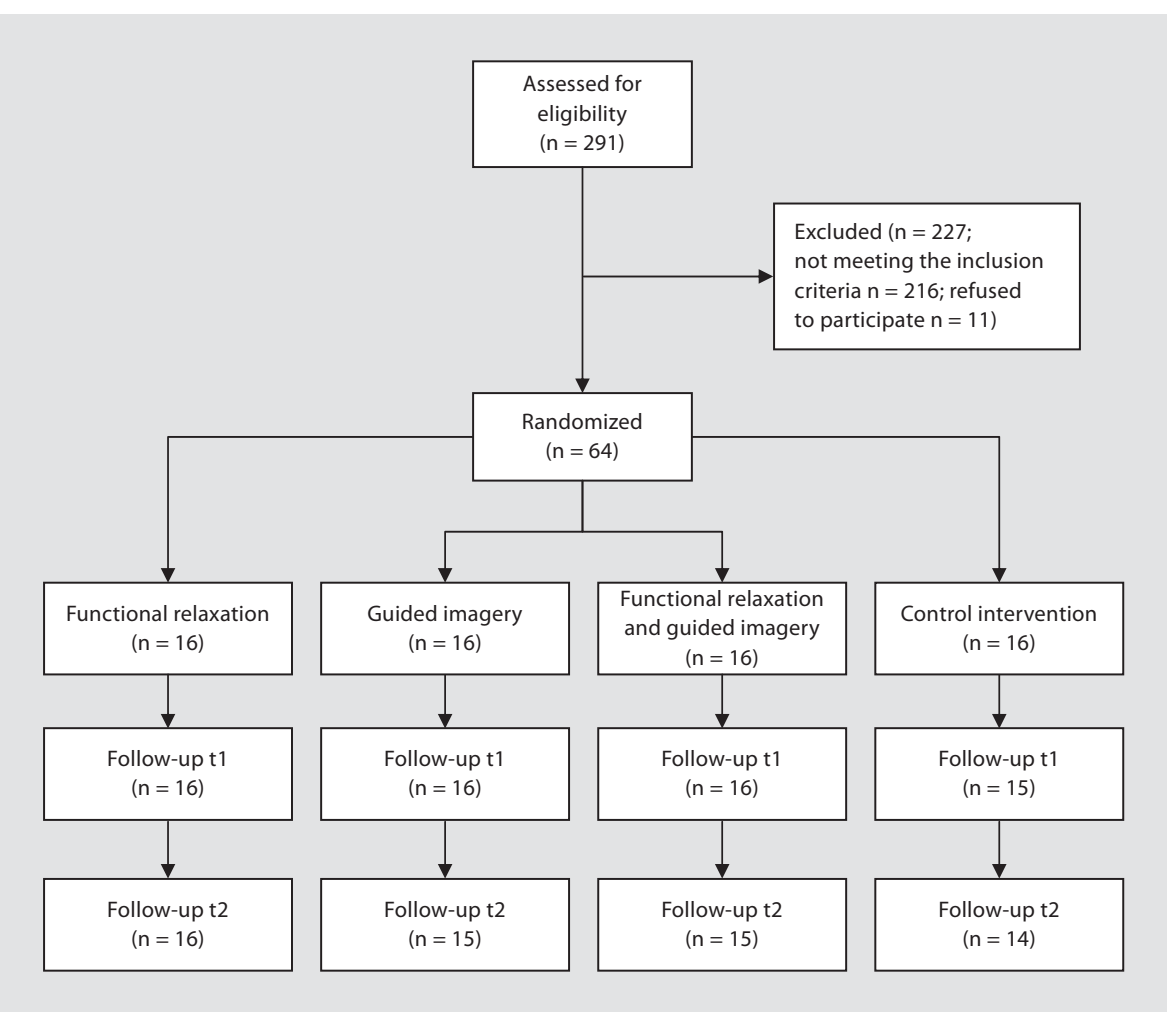

Fig. 1. Flow diagram of the trial progress.

tion was carried out in confidence by a study nurse, with allocation concealment using a randomization list created before the study.

With a sample size of 14 patients per group, the study was designed to detect differences in specific airway resistance $\left(\mathrm{sR}_{\mathrm{aw}}\right)$ change between the control and intervention groups of $130 \mathrm{kPa} / \mathrm{s}$ with $80 \%$ power, assuming a common standard deviation of 100 $\mathrm{kPa} / \mathrm{s}$ and considering an overall type I error level of 0.05 . These assumptions correspond to the results of a small pilot study. 64 patients were randomized, with a loss to follow-up of 5 patients due to incomplete or missing data.

\section{Assessment}

The standard pulmonary function indicators were measured using a spirometer and a body plethysmograph (Jaeger ${ }^{\circledR}$ ), always at the same time of the day. These included standard assessments of forced expiratory volume per second $\left(\mathrm{FEV}_{1}\right)$ as a percentage of the predicted value and the $\mathrm{sR}_{\mathrm{aw}}$, also reported as a percentage of the predicted value. The best of 3 satisfactory $F_{E V}$ and $s R_{a w}$ tests was recorded. Normal values were calculated from the sex, height and age of each patient, in order to compare the measured values with the normal values [24]. The actual value of the lung function test is calculated as a percentage of the nominal value. The evaluation considers the $\mathrm{FEV}_{1}$ and the $\mathrm{sR}_{\mathrm{aw}}$ as products of the respiratory resistance and the intrathoracic gas volume. As it is independent from patient cooperation, the $\mathrm{sR}_{\mathrm{aw}}$ is considered to be a sensitive and reliable measure of resistance [24]. An increase in the $\mathrm{FEV}_{1}$ associated with a decrease in the $\mathrm{sR}_{\mathrm{aw}}$ indicates a bronchodilatation. The average duration for a complete pulmonary func- tion test was approximately $7 \mathrm{~min}$. The data were analyzed by an online digital computer.

\section{Design}

The time structure of the trial was identical for all conditions. After giving informed consent, the patients were randomized to FR, GI, FR/GI or the CI. The interventions were carried out over a period of 4 weeks in small groups of 3-5 persons, with 1 group session per week. The participants underwent lung function tests directly before ( $\mathrm{t} 0$ ) and after finishing the therapies ( $\mathrm{t} 1$ ) and again 4 months later ( $\mathrm{t} 2$ ). The test was always taken at the same time of the day using a standardized diagnostic procedure, as recommended by the European Respiratory Society [24]. On assessment days, the patients were not allowed to take any short-acting medication before the examination, which prevented false results caused by potential contamination of effects. The study was completed according to the plan.

\section{Interventions}

All interventions were carried out by a physician specialized in psychosomatic medicine and certified in functional relaxation and hypnotherapy, who was assisted by a PhD student.

FR is a somatopsychotherapeutic intervention technique commonly used in Germany, Austria and Switzerland for the treatment of psychosomatic disorders $[25,26]$. The therapeutic effects are assumed to be delivered by positive stimulation of the autonomic nervous system as well as by facilitation of proprioreceptive awareness [27]. Minute movements of small joints, hardly noticeable to observers, are performed during relaxed expiration, 
accompanied by a focus on and exploration of the perceived differences of body feelings triggered by these movements. The focus of attention is thereby directed towards the way the person relates to the environment, particularly the floor as the foundation and as the 'outer support' to the bony skeleton (referred to in FR as 'the frame' or the 'inner support'), to the interior regions of the body and to the skin as an 'outer border'. Unlike exercise-based methods, such as progressive muscle relaxation, the bodily perceptions are explicitly verbalized and processed in the therapeutic relationship.

In the FR treatment, the group therapy was guided by a manual which was generated during previous investigations $[25,28]$. This concept has been adapted to the special features of asthmatic patients.

The GI intervention consisted of visualizing a set of standardized guided imageries targeting the airways [29]. In the first session, participants were instructed in positive visualization of a situation with deep 'free' breathing. Additionally, the patients were encouraged to integrate this positive imagination into their daydreams. The second session started with a short educational section about allergy and the relevance of mast cells, followed by an exercise to imagine the mast cells acting to alleviate the hypersensitive bronchial system. The third session focused on a healthy self-perception, including imagining a better physical condition in the future. The last session provided time for repetition of the aforementioned topics.

In the FR/GI group, the FR units were augmented by elements of imagery in each session. In contrast, the CI consisted of isotonic exercises, which required - in comparison to FR - an equivalent amount of movement, but which did not involve the chest, shoulders, neck or head. This intervention did not focus on enhancing bodily selfawareness, as in FR, neither did it include imaginative elements, as in GI. During the isotonic exercises the patients were instructed to hold specific postures for several minutes in a relaxed manner. To enhance its credibility, the patients were told that this new technique was called focused body awareness training.

In the interest of minimizing potential therapist bias, all 3 forms of intervention were conducted by the same therapeutic team. The time structure was the same in every group with one 60 -min session weekly and a total of 4 sessions over a 4 -week-period. The therapy sessions were videotaped and supervised by an independent, certified body psychotherapist, ensuring that the therapists adhered to the manual.

\section{Statistical Analysis}

Statistical analyses were performed using SPSS software v.15.0 (SPSS Inc., Chicago, Ill., USA). The patients' basic data were characterized by descriptive statistics. The $\chi^{2}$ test and analysis of variance (ANOVA) were used for exploratory comparisons of social demographic data and baseline values between intervention groups. Analysis of covariance (ANCOVA) was employed for analyzing differences in long-term change of the primary outcome parameters $\mathrm{FEV}_{1}$ and $\mathrm{sR}_{\mathrm{aw}}$ between the control and intervention groups. Baseline $\mathrm{FEV}_{1}$ and $\mathrm{sR}_{\mathrm{aw}}$ were included as adjustment covariates in order to control for the impact of initial value levels and different distributions in treatment groups. All statistical analyses were performed two-sided at a 0.05 level of significance. To adjust for multiple group comparisons, Bonferroni correction of $\mathrm{p}$ values was conducted. To minimize the required correction level in multiple test procedures, statistical comparisons of intervention groups were solely conducted for the clinically relevant changes of $\mathrm{FEV}_{1}$ and $\mathrm{sR}_{\mathrm{aw}}$ between measurement 2 and baseline. Statistical evaluation was carried out according to the intentionto-treat principle.

\section{Source of Funding and Ethical Considerations}

The study was planned and conducted in accordance with the Declaration of Helsinki and ethical laws pertaining to the medical professions. The design was approved by the ethics committee of the Statutory Physician Board. This study was conducted without any institutional influence and was not funded externally.

\section{Results}

The study sample consisted of 64 patients. The average age of all participants was $42.9 \pm 12.2$ years, $64 \%$ were female. No statistically significant group differences were found between the 3 treatment groups regarding sociodemographics (age, sex, partnership and professional status) and baseline measurements at $\mathrm{t} 0$. Tables 1 and 2 show the explorative analysis of the outcome parameters $\mathrm{FEV}_{1}$ and $\mathrm{sR}_{\mathrm{aw}}$ over the course of the trial. No statistically significant differences were found in mean baseline values. Controller or reliever medication was kept stable in all patients throughout the study. All $p$ values reported here resulted from group comparisons of changes in $\mathrm{FEV}_{1}$ and/or $\mathrm{sR}_{\mathrm{aw}}$ (from baseline to t2) with CI as a comparison group, and they are adjusted for baseline $\mathrm{FEV}_{1}$ and $\mathrm{sR}_{\mathrm{aw}}$, respectively. Bonferroni correction of $\mathrm{p}$ values was used in order to apply an overall level of significance of $5 \%$ in the face of multiple testing.

Participation in the FR, GI, FR/GI and CI groups led to increases in $\mathrm{FEV}_{1}$ (\% predicted) of $7.6 \pm 13.2,3.3 \pm$ 9.8 and $8.3 \pm 21.0$, respectively, as compared to $-1.8 \pm$ 11.1 in the CI group at the end of the therapy phase. At $\mathrm{t} 2$, the increases in $\mathrm{FEV}_{1}$ were $6.9 \pm 10.3(\mathrm{p}=0.009)$ in the FR group, $4.4 \pm 7.3(\mathrm{p}=0.072)$ in the GI, $4.5 \pm 8.1(\mathrm{p}=$ $0.066)$ in the FR/GI and $-2.8 \pm 9.2$ in the CI. While the most beneficial effect regarding $\mathrm{FEV}_{1}$ at $\mathrm{t} 1$ could be seen in patients who participated in FR/GI (followed by FR and GI alone, with a slight decrease in the CI group), a long-lasting effect at $\mathrm{t} 2$ could only be observed in the FR group, with a noticeable decrease in the benefit among FR/GI and GI patients and a further decline in the CI group. The analyses relating to changes in $\mathrm{FEV}_{1}$ revealed that only the FR group reached a level of statistical significance when compared to the CI.

Regarding changes in $\mathrm{sR}_{\mathrm{aw}}$ (\% predicted), at the end of the therapy phase ( $\mathrm{t} 1)$ the decreases in $\mathrm{sR}_{\mathrm{aw}}$ were -162.2 
Table 1. Measurement of $\mathrm{FEV}_{1}$ (\% predicted) over the course of the trial

\begin{tabular}{|c|c|c|c|c|c|c|c|c|c|c|c|c|}
\hline \multirow[t]{2}{*}{$\mathrm{FEV}_{1}$} & \multicolumn{4}{|l|}{ to } & \multicolumn{4}{|l|}{$\mathrm{t} 1$} & \multicolumn{4}{|l|}{$\mathrm{t} 2$} \\
\hline & FR & GI & FR/GI & CI & FR & GI & FR/GI & CI & FR & GI & FR/GI & CI \\
\hline Patients & 16 & 16 & 16 & 16 & 16 & 16 & 16 & 15 & 16 & 15 & 15 & 13 \\
\hline Mean & 80.2 & 80.8 & 81.8 & 82.1 & 87.8 & 84.1 & 84.9 & 80.8 & 87.1 & 85.6 & 85.7 & 76.9 \\
\hline Standard deviation & 16.1 & 17.7 & 13.5 & 16.2 & 14.5 & 15.4 & 12.2 & 19.0 & 14.5 & 15.5 & 14.0 & 19.7 \\
\hline Minimum & 54 & 40 & 54 & 51 & 45 & 47 & 59 & 40 & 64 & 55 & 60 & 35 \\
\hline Maximum & 105 & 105 & 106 & 113 & 101 & 104 & 100 & 107 & 112 & 109 & 106 & 108 \\
\hline 25th percentile & 65.8 & 72.0 & 70.0 & 72.0 & 84.0 & 71.3 & 77.5 & 70.0 & 74.0 & 73.0 & 73.0 & 71.5 \\
\hline 50th percentile & 81.0 & 82.0 & 84.0 & 80.5 & 91.5 & 86.0 & 88.0 & 83.0 & 88.5 & 89.0 & 89.0 & 78.0 \\
\hline 75th percentile & 96.5 & 94.8 & 90.0 & 92.8 & 97.5 & 97.0 & 92.5 & 97.0 & 99.5 & 98.0 & 96.0 & 92.0 \\
\hline
\end{tabular}

Table 2. Measurement of $\mathrm{sR}_{\mathrm{aw}}$ (\% predicted) over the course of the trial

\begin{tabular}{|c|c|c|c|c|c|c|c|c|c|c|c|c|}
\hline \multirow[t]{2}{*}{$\mathrm{sR}_{\mathrm{aw}}$} & \multicolumn{4}{|l|}{ to } & \multicolumn{4}{|l|}{$\mathrm{t} 1$} & \multicolumn{4}{|l|}{$\mathrm{t} 2$} \\
\hline & FR & GI & FR/GI & CI & FR & GI & FR/GI & CI & FR & GI & FR/GI & CI \\
\hline Patients & 16 & 16 & 16 & 16 & 16 & 16 & 16 & 15 & 16 & 15 & 15 & 13 \\
\hline Mean & 286.3 & 252.9 & 249.6 & 226.1 & 162.2 & 207.4 & 158.8 & 258.6 & 185.4 & 203.1 & 172.9 & 267.6 \\
\hline Standard deviation & 123.6 & 149.9 & 111.9 & 85.6 & 56.6 & 87.9 & 93.3 & 112.4 & 71.3 & 117.5 & 91.5 & 94.3 \\
\hline Minimum & 104 & 100 & 93 & 89 & 70 & 110 & 62 & 119 & 101 & 85 & 57 & 127 \\
\hline Maximum & 512 & 638 & 494 & 443 & 271 & 372 & 450 & 531 & 382 & 517 & 379 & 436 \\
\hline 25 th percentile & 193.3 & 129.5 & 150.8 & 166.5 & 128.5 & 138.8 & 92.5 & 198.0 & 136.8 & 112.0 & 100.0 & 204.5 \\
\hline 50th percentile & 243.0 & 212.5 & 254.0 & 209.5 & 149.5 & 174.5 & 145.0 & 215.0 & 170.5 & 185.0 & 167.0 & 229.0 \\
\hline 75th percentile & 405.8 & 348.8 & 314.0 & 285.8 & 180.5 & 251.5 & 195.8 & 302.0 & 216.8 & 216.0 & 232.0 & 365.0 \\
\hline
\end{tabular}

\pm 56.6 in the FR group, $-207.4 \pm 87.9$ in the GI, -158.8 \pm 93.3 in the FR/GI and $-265.3 \pm 111.5$ in the CI. After the 4-month follow-up ( $\mathrm{t} 2$ ), the decreases in $\mathrm{sR}_{\mathrm{aw}}$ were $-100.9 \pm 100.5(\mathrm{p}=0.018)$ in the FR group, $-51.7 \pm 165.2$ $(\mathrm{p}=0.228)$ in the GI, $-72.4 \pm 56.5(\mathrm{p}=0.024)$ in the FR/ GI and $54.9 \pm 86.2$ in the CI. At t 1 the clearest positive effect in $\mathrm{sR}_{\mathrm{aw}}$ could be seen with FR, followed by FR/GI and GI alone, whereas even a minor aggravation of airway resistance could be observed in the group receiving the CI. This pattern was equal at $\mathrm{t} 2$, with a marginal decrease over time in the FR, FR/GI and GI groups and a slightly exacerbated aggravation in patients receiving the CI. Group comparisons revealed that improvements in the FR and FR/GI groups, but not in the GI group, reached a 0.05 level of significance at $t 2$.

No side effects from the interventions were reported by any of the patients, nor did any patients report difficulties with implementing the body-psychotherapeutic technique of FR.

Relaxation and Guided Imagery in Asthma

\section{Discussion}

This study confirms earlier findings showing a positive effect from FR on lung parameters in patients with bronchial asthma [20, 21].

In the present study we could demonstrate for the first time that effects achieved through FR remain stable at a 4-month follow-up. While improvements in the GI group initially seemed clinically relevant, they dissipated significantly more rapidly during the follow-up observation period. The effects of the combined FR/GI intervention fell in between those achieved in the other treatment groups. One could speculate that this differential effect is due to the active and individual FR approach, which allows the patient to further apply individually modified exercise elements to various everyday situations over the course of the therapy phase and catamnesis.

Little can be said regarding the therapeutic mechanisms involved as no relevant information is available yet regarding the psychophysiological effects of body-orient- 
ed therapy or imagery techniques in asthma. As all patients included in the present trial suffered from allergic, and therefore immunoglobulin-E (IgE)-mediated asthma, the effects may be partially due to a modulation of allergic antibody responses. Castés et al. [30] demonstrated that a 6-month psychosocial intervention program, including relaxation, GI and self-esteem training, led not only to a clinically significant improvement, but also to a significant reduction in the specific IgE responses against Ascaris lumbricoides (the most important allergen in the children in that study). Beyond potential modulation of the IgE-mediated inflammatory response, the observed benefits could be partially due to direct bronchodilatatory effects caused by FR or GI. The underlying mechanism might be a mitigation of vagal stimulation, as 'rebalancing' of the autonomic nervous system is assumed to be a possible mode of action of FR. Furthermore, the coincident reduction of asthma-associated anxiety presumably accounts for improvements of lung function, as shown by Lolak et al. [31].

The deterioration of $\mathrm{sR}_{\mathrm{aw}}$ and $\mathrm{FEV}_{1}$ over time in the CI group might be explained by a negative placebo expectation, a so-called nocebo effect. The nocebo hypothesis proposes that expectations of sickness and the affective states associated with such expectations can cause sickness in the expectant patient [32]. All participants were informed about the possibility of being randomized to any 1 of the 3 different treatment settings (FR, GI, FR/GI) or to the control group. As the CI consisted only of an instruction of specific bodily postures without any specific movements or pictures for imagination, this intervention was potentially unmasked as a placebo intervention by a considerable proportion of participants. This might have led to a more pessimistic attitude towards the effectiveness of the intervention, and pessimists are known to be more likely to display a negative placebo (nocebo) expectation [33].

This study has several methodological limitations. First, the sample size was relatively small, consisting of only 16 patients in each treatment condition. Since the follow-up period of this trial was only 4 months, the dropout rate might have been lower than in trials with a longer follow-up period.

Given that the body-oriented therapy sessions were carefully designed and matched for possible nonspecific effects (e.g. attention given by the trainer or learning effects), it is difficult to claim that the outcome variables were influenced by nonspecific components of the FR treatment. However, one possible influence may be that the study was not conducted in an entirely double-blind setting. However, creating completely double-blind conditions would not have been feasible, since it is impossible to prevent an experienced therapist knowing the kind of technique she/he is applying. However, a potential bias could arise from not checking the blinding of the patients. Although patients were not informed of which intervention they were allocated to, we did not ask the participants which intervention they believed themselves to be receiving. We also did not measure the degree of subjectively perceived relaxation in the FR group or the degree of vividness in the GI group. Although adherence of therapists to the treatment manual was controlled by continuous supervision, the adherence of patients to the given instructions was not measured in the trial.

The present trial focused on the influence of FR, GI and FR/GI on lung parameters in patients with allergic asthma and, therefore, no subjective outcome parameters were measured. It has to be considered that improved physiological outcomes are not necessarily reflected in an improved asthma-related quality of life [34].

Even considering the above limitations, the results of our study are promising because they show that bodyoriented therapy, such as FR, can lead to a significant reduction in the objective parameters of pulmonary obstruction in asthma which, furthermore, was shown to be maintained over a period of 4 months. These treatment benefits are the result of only 4 sessions of complementary body-oriented therapy, which can, therefore, be regarded as a feasible intervention that is an efficient use of time. Nevertheless, future research should consider further the feasibility of body-oriented therapy in daily practice. Additional research is needed to ascertain if these results can be replicated, for how long the benefits last beyond a 4-month follow-up and if the regular complementary use of FR leads to clinically relevant improvements in the long-term course, including improvement in health-related quality of life.

\section{Acknowledgements}

The authors thank Silke Witt-Wulff, MD, for her support in the trial, and Mike Cronan, MA, for his linguistic revision of the manuscript. 


\section{References}

1 ECRHS: Variations in the prevalence of respiratory symptoms, self-reported asthma attacks, and use of asthma medication in the European Community Respiratory Health Survey (ECRHS). Eur Respir J 1996;9:687695.

2 ISAAC: Worldwide variations in the prevalence of asthma symptoms. The International Study of Asthma and Allergies in Childhood (ISAAC). Eur Respir J 1998;12:315335.

3 Eder W, Ege MJ, von Mutius E: The asthma epidemic. N Engl J Med 2006;355:22262235.

4 Lahmann C, Loew T, Leiberich P, Nickel M, Pfeifer M: Psychosomatic medicine in pneumology (in German). Psychodyn Psychother 2005;4:106-114.

5 Lehrer PM: Emotionally triggered asthma: a review of research literature and some hypotheses for self regulation therapies. Appl Psychophysiol Biofeedback 1998;23:13-41.

6 Mathé AA, Knappe PH: Emotional and adrenal reactions to stress in bronchial asthma. Psychosom Med 1971;33:323-340.

7 Aboussafy D, Campbell TS, Lavoie K, Aboud FE, Ditto B: Airflow and autonomic responses to stress and relaxation in asthma: the impact of stressor type. Int J Psychophysiol 2005;57:195-201

8 Leigh D, Groen J, Rees L, Wretmark G, Wolff HG: Reproducible psychogenic attacks of asthma. J Psychosom Res 1956;1:58-67.

9 Steptoe A, Holmes R: Mood and pulmonary function in adult asthmatics: a pilot selfmonitoring study. Br J Med Psychol 1985;58: 87-94.

10 Yorke J, Fleming SL, Shuldham C: Psychological interventions for adults with asthma: a systematic review. Respir Med 2007;101: $1-14$.

11 Vamos M, Kolbe J: Psychological factors in severe chronic asthma. Aust NZ J Psychiatry 1999;33:538-544.

12 Huntley A, White AR, Ernst E: Relaxation therapies for asthma: a systematic review. Thorax 2002;57:127-131.

13 Nickel C, Lahmann C, Muehlbacher M, Pedrosa Gil F, Kaplan P, Buschmann W, Tritt K, Kettler C, Bachler E, Egger C, Anvar J, Fartacek R, Loew T, Rother W, Nickel M: Pregnant women with bronchial asthma benefit from progressive muscle relaxation: a randomized, prospective, controlled trial. Psychother Psychosom 2006;75:237-243.
14 Ewer OC, Stewart DE: Improvement in bronchial hyper-responsiveness in patients with moderate asthma after treatment with a hypnotic technique: a randomised controlled trial. BMJ 1986;293:1129-1132.

15 Henry M, de Rivera JLG, Gonzalez-Martin IJ, et al: Improvement of respiratory function in chronic asthmatic patients with autogenic therapy. J Psychosom Res 1992;17:265-270.

16 Coen BL, Philip B, McGrady A, et al: Effects of biofeedback-assisted relaxation on asthma severity and immune function. Pediatr Asthma Allergy Immunol 1996;10:71-78.

17 Manocha R, Marks GB, Kenchington P, Peters D, Salome CM: Sahaja yoga in the management of moderate to severe asthma: a randomised controlled trial. Thorax 2002;57: 110-115.

18 Brown D: Evidence-based hypnotherapy for asthma. A critical review. Int J Clin Exp Hypn 2007;55:220-249.

19 Freeman LW, Welton D: Effects of imagery, critical thinking, and asthma education on symptoms and mood state in adult asthma patients: a pilot study. J Altern Complement Med 2005;11:57-68.

20 Loew T, Siegfried W, Martus P, Tritt K, Hahn EG: Functional relaxation reduces acute airway obstruction in asthmatics as effectively as inhaled terbutaline. Psychother Psychosom 1996;65:124-128.

21 Loew TH, Tritt K, Siegfried W, Bohmann H, Martus P, Hahn EG: Efficacy of 'functional relaxation' in comparison to terbutaline and a 'placebo relaxation' method in patients with acute asthma. A randomized, prospective, placebo-controlled, crossover experimental investigation. Psychother Psychosom 2001;70:151-157.

22 Moher D, Schulz KF, Altman DG, the Consort Group: The Consort statement: revised recommendations for improving the quality of reports of parallel-group randomised trials. Lancet 2001;357:1191-1194.

23 Global Initiative for Asthma: Global Strategy for Asthma Management and Prevention. 2006. www.ginasthma.org.
24 Quanjer PH, Tammeling GJ, Cotes JE, Pedersen OF, Peslin R, Yernault JC: Lung volumes and forced ventilatory flows. Eur Respir J Suppl 1993;16:5-40.

25 Lahmann C, Loew TH, Tritt K, Nickel M: Efficacy of functional relaxation and patient education in the treatment of somatoform heart disorders: a randomized, controlled clinical investigation. Psychosomatics 2008; 49:378-385

26 Lahmann C, Schoen R, Henningsen P, Ronel J, Muehlbacher M, Loew T, Tritt K, Nickel M, Doering S: Brief relaxation versus music distraction in the treatment of dental anxiety. A randomized controlled clinical trial. J Am Dent Assoc 2008;139:317-324.

27 Arnim A: Functional relaxation - psychotherapeutic tutorial. Fundamenta Psychiatrica 1994;8:196-203.

28 Loew TH, Sohn R, Martus P, Tritt K, Rechlin T: Functional relaxation as a somatopsychotherapeutic intervention: a prospective controlled study. Altern Ther Health Med 2000; 6:70-75.

29 Rietveld S, Everaerd W, van Beest I: Excessive breathlessness through emotional imagery in asthma. Behav Res Ther 2000;38: 1005-1014.

30 Castés M, Hagel I, Palenque M, Canelones P, Corao A, Lynch NR: Immunological changes associated with clinical improvement of asthmatic children subjected to psychosocial intervention. Brain Behav Immun 1999;13: $1-13$.

31 Lolak S, Connors GL, Sheridan MJ, Wise TN: Effects of progressive muscle relaxation training on anxiety and depression in patients enrolled in an outpatient pulmonary rehabilitation program. Psychother Psychosom 2008;77:119-125.

32 Hahn RA: The nocebo phenomenon: concept, evidence, and implications for public health. Prev Med 1997;26:607-611.

33 Geers AL, Kosbab K, Helfer SG, Weiland PE, Wellman JA: Further evidence for individual differences in placebo responding: an interactionist perspective. J Psychosom Res 2007; 62:563-570.

34 Carranza Rosenzweig JR, Edwards L, Lincourt W, Dorinsky P, Zu Wallack RL: The relationship between health-related quality of life, lung function and daily symptoms in patients with persistent asthma. Respir Med 2004;98:1157-1165. 